JOURNAL OF PHYSICS AND CHEMISTRY OF SOLIDS

\title{
Influence of electron diffraction on measured energy-resolved momentum densities in single-crystalline silicon
}

\author{
M. Vos*, A.S. Kheifets, V.A. Sashin, E. Weigold \\ Atomic and Molecular Physics Laboratories, Research School of Physical Sciences and Engineering, The Australian National University, \\ Canberra ACT 0200, Australia
}

Received 6 April 2003; revised 13 August 2003; accepted 22 August 2003

\begin{abstract}
Electron momentum spectroscopy is used to determine the spectral function of silicon single crystals. In these experiments $50 \mathrm{keV}$ electrons impinge on a self-supporting thin silicon film and scattered and ejected electrons emerging from this sample with energies near $25 \mathrm{keV}$ are detected in coincidence. Diffraction effects are present that give rise to additional structures in the measured spectral momentum densities. Spectra for a specific momentum value can be obtained at different orientations of the crystal relative to the analysers. By comparing these spectra for which the measured momentum density is the same, but the diffraction conditions of the incoming and outgoing electron trajectories differ, one can distinguish between features due to diffraction of the incoming and/or outgoing electrons, and those due to the electronic structure of the target itself.

(C) 2003 Elsevier Ltd. All rights reserved.
\end{abstract}

PACS: 71.20.Mq; 79.20.Kz; 61.14. - $\mathrm{x}$

Keywords: C. Electron; diffraction

\section{Introduction}

For crystalline solids the electronic structure is usually described by Bloch functions $\psi_{\varepsilon, \mathbf{q}}(\mathbf{r})=\sum_{\mathbf{G}} c_{\mathbf{G}} \mathrm{e}^{\mathrm{i}(\mathbf{G}+\mathbf{q}) \cdot \mathbf{r}}$, where $\mathbf{q}$ is the crystal momentum, chosen in the first Brillouin zone, and the sum extends over all reciprocal lattice vectors G. A lot of experimental work (e.g. angle-resolved photoemission [1]) is available dealing with the dispersion, i.e. the change in energy $\varepsilon$ as a function of q. Very little experimental work deals with the magnitude of the contributions of different plane waves to the Bloch function.

Electron momentum spectroscopy (EMS) can in principle measure the energy-resolved momentum densities [2] and hence determine the contribution $\left|c_{G}\right|^{2}$ of the different plane waves components to the Bloch function. In these kinematically complete scattering experiments an incoming electron beam with a precisely known energy $\left(E_{0}\right)$ and momentum $\left(\mathbf{k}_{0}\right)$ impinges on a target. Some of the incoming particles are

\footnotetext{
* Corresponding author. Tel.: + 61-2-6125-4985; fax: +61-6125 2452.

E-mail address: maarten.vos@anu.edu.au (M. Vos).
}

scattered in a binary electron-electron collision and a target electron is ejected. By measuring the energies $\left(E_{1}, E_{2}\right)$ and momenta $\left(\mathbf{k}_{1}, \mathbf{k}_{2}\right)$ of the scattered and ejected electron in coincidence one can, using elementary conservation laws, determine the energy and momentum transferred to the target. The energy-momentum distribution obtained in this way is proportional to the spectral function of the target.

This technique applies both to (gas-phase) atomic and molecular targets as well as to solids [3]. For gas-phase targets (where molecules are present in all possible orientations) or polycrystalline thin film targets one obtains a spherically averaged spectral function, while for singlecrystalline targets the directional anisotropy of the electronic structure can be investigated. Single crystal EMS data are scarce. They are confined to graphite $[4,5]$ and silicon $[6$, 7]. The published silicon data, obtained with the Flinders University EMS spectrometer, are difficult to interpret due to high levels of multiple scattering. For the present measurements, using the high-energy bulk-sensitive EMS spectrometer of the Australian National University [8], multiple scattering is much reduced and these data reveal a plethora of details obscured in the previous measurement. 
Unfortunately for EMS measurements on films of finite thickness, elastic multiple scattering effects contaminate the data. For crystalline materials the contribution of elastic scattering from different atoms add up coherently (electron diffraction) and this causes additional structures $[9,10]$. The change of the incoming or outgoing electron momenta by diffraction changes the outcome of the measurement by a reciprocal lattice vector. Thus the diffracted intensity could be attributed to an incorrect plane wave and diffraction appears to prevent us from reaching our goal: the determination of the amplitude of different plane waves to the Bloch function. In this paper we demonstrate that different measurements can be used to disentangle the diffracted contribution and the primary (non-diffracted) contribution. In this way we can establish experimentally, at least in principle, the electronic structure in the extended zone scheme, free from coherent elastic multiple scattering effects.

\section{Experimental procedure}

\subsection{Sample preparation}

EMS experiments require extremely thin free-standing films. The initial part of the sample preparation followed the procedure described by Utteridge et al. [11]. Briefly, a buried silicon oxide layer was formed by ion implantation in a crystal with $\langle 1,0,0\rangle$ surface normal. Using wet chemical etching techniques a crater was etched in the back side of the Silicon crystal. The etching rate drops to zero when the oxide layer is reached. The silicon oxide layer is then removed by an HF dip, and the sample (thickness of the thin part $\simeq 200 \mathrm{~nm}$ ) is transferred to the vacuum. Here we use low energy $(600 \mathrm{eV})$ argon sputtering to further thin the sample. An incandescent light is positioned behind the sample. The thin part is completely transparent. However. the colour of the transmitted light changes with thickness due to interference of directly transmitted light and that reflected from the front and back silicon-vacuum interface. In this way we monitor the thinning process. It is stopped when a thickness of $20 \mathrm{~nm}$ is reached (corresponding to a greenish colour). This sample is measured without any further treatment. A thin amorphous layer of Si could be present at the backside (sputtering side) whereas the front side (facing the analysers) is probably hydrogen terminated as a consequence of the HF dip.

\subsection{Spectrometer description}

The measurements were done using the $50 \mathrm{keV}$ symmetric electron momentum spectrometer at the Australian National University. It is described extensively elsewhere [8, 12]. The spectrometer uses two-dimensional (energy and angle resolving) electrostatic energy analysers to measure efficiently the energy-resolved momentum densities.
The spectrometer is sketched in Fig. 1. The $z$-axis is taken along the incoming electron beam direction. Electrons emerging at polar angles $\Theta_{s}$ near $45^{\circ}$ and with azimuthal angles $\phi_{1,2}$ within $\pm 5^{\circ}$ with respect to the $x-z$ plane are detected by the analysers. Using this geometry, the recoil momentum of the target is zero if all three electrons (the incoming and both outgoing (scattered and ejected) electrons) are in one plane (i.e. in a single-particle picture one scattered from a stationary electron). If the momentum of the ejected electron is not in the plane defined by the trajectories of the incoming and scattered electron then the recoil momentum of the target is non-zero and is directed approximately along the $y$-axis (i.e. in a single electron picture one scattered from an electron with momentum $\left.\left(p_{x} \simeq 0, p_{y} \neq 0, p_{z} \simeq 0\right)\right)$. By aligning a specific direction of

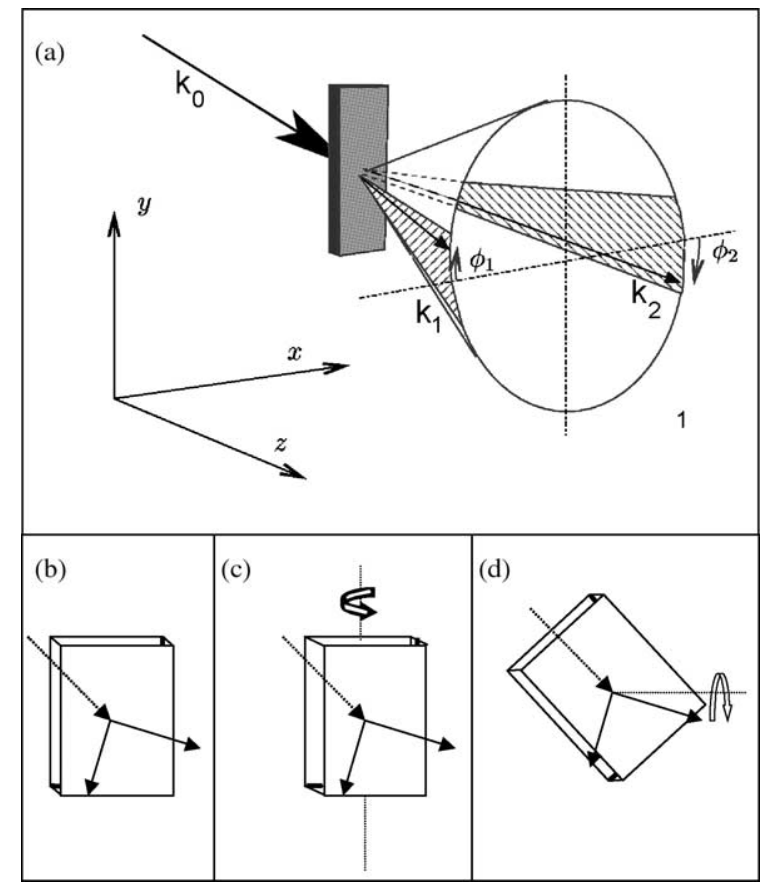

Fig. 1. In (a) we show a schematic representation of the measurement. Incoming electrons (momentum $\mathbf{k}_{0}$ ) impinge on a thin film and two analysers, measuring simultaneously a series of azimuthal angles $\phi_{1,2}$ and energies $E_{1,2}$, select coincident pairs of emerging electrons with momentum $\mathbf{k}_{1}, \mathbf{k}_{2}$. The sample is indicated as a block with the edges parallel to the $[0,1,0]$ and $[0,0,1]$, crystal directions. The scattering geometry is chosen in such a way that recoil momentum of detected coincidence events $\mathbf{k}_{0}-\mathbf{k}_{1}-\mathbf{k}_{2}$ is directed along the $y$-direction and its magnitude is proportional to $\phi_{1}-\phi_{2}$. Thus in (b) we measure the spectral momentum density along the $[0,1,0]$ direction and the incoming beam is directed along $[0,0,1]$. The outgoing electrons are moving close to $[ \pm 1,0,1]$ directions. In (c) we measure the spectral momentum density along the same direction, but the crystal has been rotated along the $y$-axis by $10^{\circ}$ moving the incoming and outgoing electrons away from high-symmetry directions. In (d) the crystal was rotated along the $[0,0,1]$ axis by $45^{\circ}$ and the spectral momentum density along a $\langle 1,1,0\rangle$ direction is measured. 
a thin crystal with the spectrometer $y$-direction we can measure the energy-resolved momentum density along that crystal direction. In practice, the actual orientation of the target can be determined by observing the diffraction pattern of the transmitted electron beam on a phosphorus screen. The sample orientation can be changed without removing the sample from the vacuum chamber (operating pressure $2-3 \times 10^{-10}$ torr).

At the high energies used in this spectrometer small deviations of $\Theta_{s}$ cause significant shifts of the line parallel to the $p_{y}$ direction, in momentum space, along which one measures the momentum (further referred to as 'measurement line'). For example a deviation of $0.1^{\circ}$ in the alignment of the collimator of the incoming beam causes the measurement line to shift by 0.1 a.u. away from $\left(p_{x}=0, p_{z}=\right.$ 0). (1 a.u. of momentum corresponds to $1.89 \AA^{-1}$.) Small magnetic fields present near the sample (in spite of the $\mu$ metal screen surrounding the spectrometer) have similar effects as small mechanical misalignments. It is therefore essential to check and, if required, correct the alignment. In practice this is done by two sets of deflector plates. Applying voltages to a set has the same effect as changing the scattering angle $\Theta_{s}$ of one of the analysers, as explained in Ref. [8]. By applying voltages corresponding to a momentum shift of $\simeq$ 0.4 a.u. to a deflector, and repeating this experiment with the opposite polarity we can measure the momentum densities shifted away from $\left(p_{x}=0, p_{z}=0\right)$ in a symmetric fashion. This is all done under computer control, changing every minute the deflector potential and collecting four different sets of spectra under otherwise identical conditions. If the alignment was perfect then all four spectra measured with the deflector voltages applied would be identical. In practise they were found to be noticeably different. The initial deviation of the measurement line from the origin was 0.2 a.u. Guided by the difference in binding energies of the quasi-particle peaks, as measured with deflector voltages applied, we estimate which deflector voltages would correspond to perfect alignment. After a few iterations of the above procedure we reached the perfect alignment within 0.03 a.u.

This alignment procedure turned out to be essential for obtaining meaningful comparison with theory. The measurements took more than three weeks. The alignment procedure was repeated at the end of the series of measurements and no significant change was found. No change in the sample quality could be inferred from the EMS data, showing that the surface condition was stable and radiation damage insignificant.

\section{The electronic structure of silicon}

\subsection{EMS without diffraction}

We briefly review the electronic structure of silicon here, emphasising the facts that are required to understand the measurements. The main aim is to avoid any confusion about what EMS measures and how it relates to the band structure as presented in the reduced zone scheme. The band structure and momentum densities as calculated by Kheifets et al. [13] are presented in Fig. 2. The band structure calculation (a full-potential linear-muffin-tin orbital calculation, based on the local density approximation to the density functional theory) provides us with a wave function in coordinate space (in the form of Bloch function $\psi_{\varepsilon, \mathbf{q}}$ $(\mathbf{r})=\sum_{\mathbf{G}} c_{\mathbf{G}} \mathrm{e}^{\mathrm{i}(\mathbf{G}+\mathbf{q}) \cdot \mathbf{r}}$, that can be characterised by a reduced momentum $\mathbf{q}$ which belongs to the first Brillouin zone), i.e. a sum of plane waves. Fourier transform of this function gives us the wave function in momentum space. Thus for each eigenvalue in energy $\varepsilon$ and reduced momentum $\mathbf{q}$ the momentum space representation is a set of delta functions.

$\phi_{\varepsilon, \mathbf{q}}(\mathbf{p})=\sum_{\mathbf{G}} c_{\mathbf{G}} \delta(\mathbf{p}-\mathbf{q}-\mathbf{G})$

where the sum is over all reciprocal lattice vectors.

The band with largest binding energy is marked 1 in Fig. 2 (top panel). The band is periodic in $k$-space having a maximum in binding energies at the $\Gamma$ points. However, the only $\Gamma$ point with significant momentum density in band 1 is the one corresponding to zero momentum. (see Fig. 2, lower panel). Thus the wave function with the lowest energy $\varepsilon$ is a Bloch function with $\mathbf{q}=0,\left|c_{(0,0,0)}\right| \simeq 1$, and the other $c_{\mathrm{G}} \simeq 0$.

By inspecting the Brillouin zone of a silicon lattice (see Fig. 3) we see that the next $\Gamma$ point (next closest to the origin) is at $\langle 1,1,1\rangle$ (1.06 a.u away from origin) (and eight symmetrically equivalent points in other quadrants) followed by $\langle 2,0,0\rangle$ (1.22 a.u. away from origin). Plane waves with these wave vectors have comparable kinetic energy and hence the lattice potential causes considerable mixing of these states. For silicon the lowest state that can be formed by a linear combination of these two types of vectors coincides with the highest occupied state (top of the valence band). Thus here we have for the top of the valence band $\mathbf{q}=0, c_{\langle 1,1,1\rangle} \neq 0, c_{\langle 2,0,0\rangle} \neq 0$ and all other $c_{\langle x, y, z\rangle} \simeq 0$.

In the experiment we measure real momentum. Thus at the bottom of the valence band we measure intensity at $\mathbf{p}=0$ and the intensity will be proportional to $\left|c_{\langle 0,0,0\rangle}\right|^{2} \simeq 1$. Now if we orient the crystal in such a way that a $\langle 2,0,0\rangle$ direction coincides with the spectrometer $y$-axis, then we measure at $\left|p_{y}\right|=1.22$ a.u. an intensity that is proportional to $\left|c_{\langle 2,0,0\rangle}\right|^{2}$. If we would orient a $\langle 1,1,1\rangle$ crystal direction with the spectrometer $y$-axis (currently not possible due to a lack of tilt motion in the manipulator) we would measure at the same binding energy, corresponding to the top of the band the intensity of $\left|c_{\langle 1,1,1\rangle}\right|^{2}$ near $p_{y}=1.06$ a.u.

Thus at $\Gamma$ the occupied part of the electronic structure of silicon is extremely simple. The bottom of the band has intensity only at $\mathbf{p}=0$. The top of the valence band has contributions only at the reciprocal lattice vectors of type $\langle 1,1,1\rangle$ and $\langle 2,0,0\rangle$. At all other reciprocal lattice points the momentum density is, for all practical purposes, zero. 

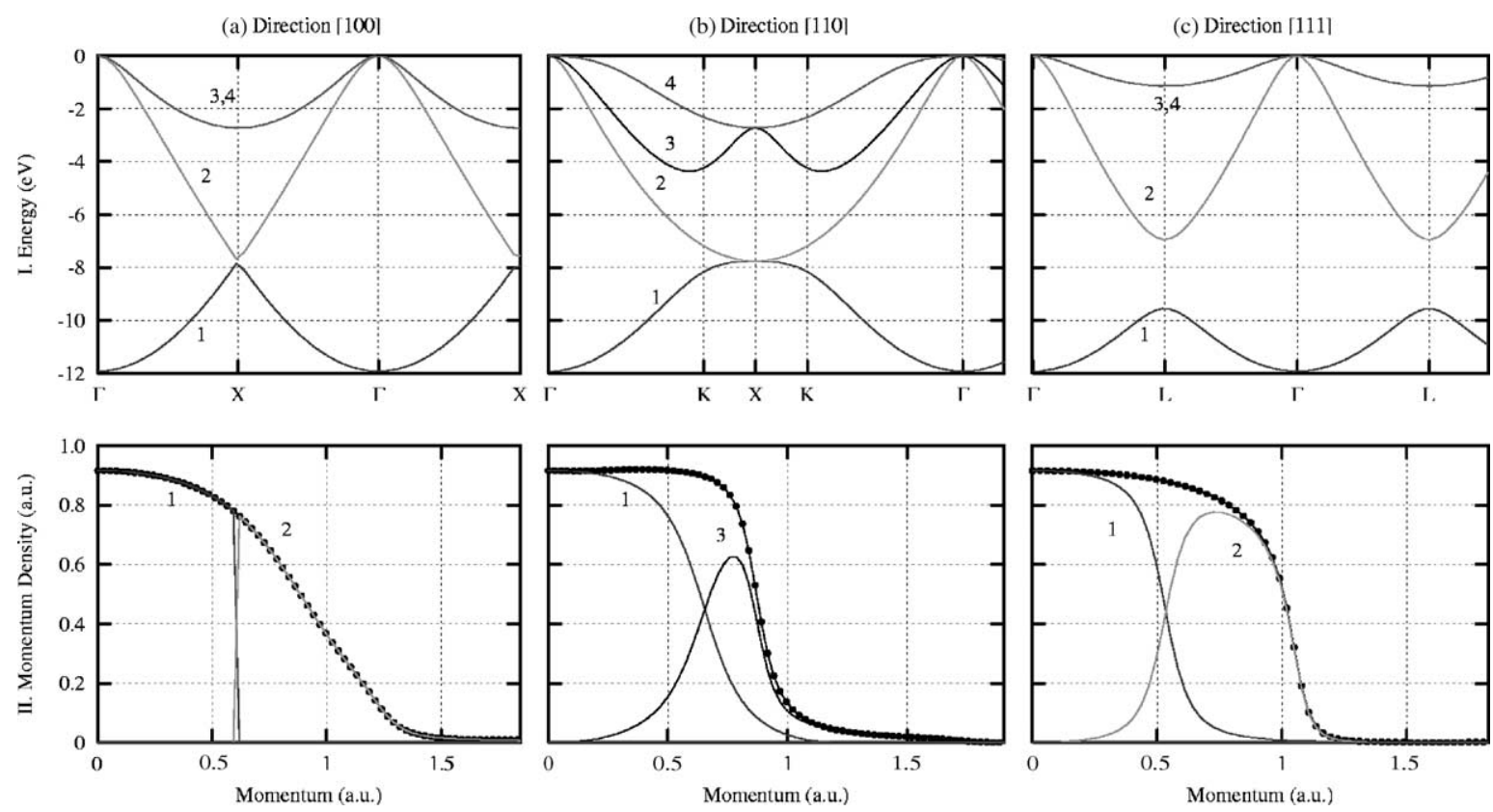

Fig. 2. Theoretical estimate of the dispersion (top panel) and momentum density (bottom panel) of Si for different crystallographic directions.

\subsection{EMS with diffraction}

Here we want to discuss qualitatively how EMS is influenced by diffraction. We assume that the effect of diffraction is just that the momentum of the incoming and/or outgoing electrons changes by a reciprocal lattice vector. As discussed in Section 5 this is an oversimplification, as the initial and diffracted beams have a well-defined phase relation. However, we have not yet seen any experimental data that cannot be described by this simplified model. For a complete quantitative, theoretical analysis see earlier published work $[9,6,10]$. To keep the language simple we assume that the target electrons are independent particles each with a well defined momentum $p_{\mathrm{e}}$.

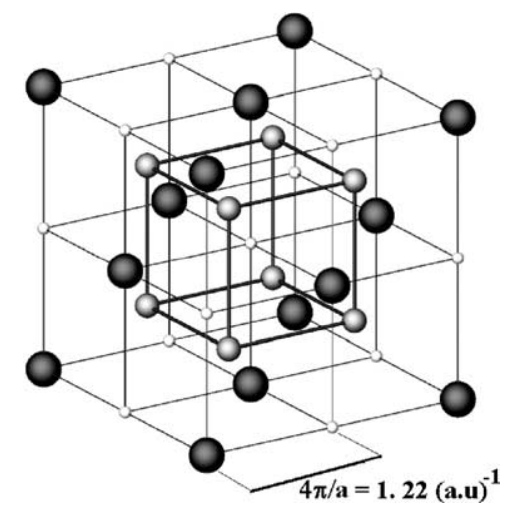

Fig. 3. The reciprocal lattice of silicon, is a body-centered cube with side $4 \pi / a$. The reciprocal lattice vectors are defined as multiples of $2 \pi / a$. Small spheres do not contribute to diffraction.
Generalisation to an interacting electron gas is straightforward. Without diffraction the momentum of the ejected electron before the collision $p_{\mathrm{e}}$ is equal to the observed momentum $p_{\mathrm{obs}}$ and is given by momentum conservation:

$p_{\mathrm{obs}}=p_{\mathrm{e}}=\mathbf{k}_{1}+\mathbf{k}_{2}-\mathbf{k}_{0}$.

In principle diffraction can affect both the incoming and outgoing electrons. In that case we have a change of momentum of either the incoming or outgoing electrons. The observer, however, has no way of knowing that diffraction occurred. Hence the observed momentum $p_{\text {obs }}$ does not correspond to the actual electron momentum $p_{\mathrm{e}}$ anymore, and

$\mathbf{p}_{\mathrm{e}}=\left(\mathbf{k}_{1}+\mathbf{G}_{1}\right)+\left(\mathbf{k}_{2}+\mathbf{G}_{2}\right)-\left(\mathbf{k}_{0}+\mathbf{G}_{0}\right)$,

$\mathbf{p}_{\mathrm{obs}}=\mathbf{k}_{1}+\mathbf{k}_{2}-\mathbf{k}_{0}$.

Here $\mathbf{G}_{0}$ is a reciprocal lattice vector corresponding to the change in momentum of the incoming electron due to diffraction, with similar meanings for $\mathbf{G}_{1}$ and $\mathbf{G}_{2}$. As our sample is thin $(20 \mathrm{~nm})$ compared to the extinction length, $\mathbf{G}_{0}, \mathbf{G}_{1}$ and $\mathbf{G}_{2}$ will correspond to $(0,0,0)$ for most coincidences. In this paper $\mathbf{p}_{\mathrm{obs}}$ is always directed along the $y$-axis and hence we use notations like $p_{\text {obs }}=y$ as a shorthand for $\mathbf{p}_{\text {obs }}=(0, y, 0)$.

For simplicity, we consider first the case for the crystal oriented such that the $[1,0,0],[0,1,0]$ and $[0,0,1]$ crystallographic directions coincide, respectively, with the $x, y$ and $z$ axes of the spectrometer (see Fig. 1(b)). As diffraction is an elastic effect, it cannot change the magnitude of $\mathbf{k}_{0}$ (or $\mathbf{k}_{1}$ or $\mathbf{k}_{2}$ ). Electron momenta are very large in the present experiment: $k_{0}=62.1$ a.u. for $E_{0}=50 \mathrm{keV}$ and $k_{1,2}=$ 43.4 a.u. for $E_{1,2}=25 \mathrm{keV}$. This means that for the most 
intense diffraction the vectors $\mathbf{G}_{i}$ are much smaller than $k_{i}(i=0,1,2)$ and therefore $\mathbf{G}_{i}$ has to be close to perpendicular to $\mathbf{k}_{i}$ to fulfill the diffraction condition $2 \mathbf{k}_{i}$. $\mathbf{G}_{i}+G_{i}^{2}=0$. (As the sample is not expected to be perfectly flat over the area of the electron beam $(0.1 \mathrm{~mm}$ diameter $)$ the Bragg condition will be fulfilled for part of the crystal if $2 \mathbf{k}_{i} \cdot \mathbf{G}_{i}+G_{i}^{2} \simeq 0$ )

Let us first focus on diffraction of the incoming beam. Its momentum $\mathbf{k}_{0}$ is aligned with the surface normal of the crystal (the $[0,0,1]$ direction) and thus $\mathbf{G}_{0}$ is restricted to $[i, j, 0]$ type reciprocal lattice vector. The reciprocal lattice vector with smallest magnitude is $[ \pm 2,0,0]$ (or $[0, \pm 2,0]$ ). However, due to the arrangement of the two silicon atoms in the primitive unit cell the diffraction intensity of these reciprocal lattice vectors (and all other vectors indicated by small spheres in Fig. 3) is 0 (see e.g. [14]). Hence, the smallest lattice vectors contributing to diffraction are of type $[ \pm 2, \pm 2,0]$. Without diffraction we measure the momentum density along the $p_{y}$-axis, more specifically the measurement line extends from $\mathbf{p}_{\text {obs }}=\mathbf{p}_{\mathrm{e}}=(0,-5,0)$ a.u. to $(0,5,0)$ a.u. (which momentum is observed depends on the azimuthal angles $\phi_{1,2}$ of the detected electrons). As is clear from the bottom panels of Fig. 2 the main electron density is restricted to momentum values smaller than 1.25 a.u. Thus, without diffraction, intensity will be only observed for $\mathbf{p}_{\text {obs }} \mid \leq 1.25$ a.u. If, for example, a [2,2,0] diffraction event has occurred the measurement line changes to the line from $\mathbf{p}_{\mathrm{e}}=(-1.22,(-5-1.22), 0)$ a.u. to $\mathbf{p}_{\mathrm{e}}=(-1.22,(5-$ $1.22), 0)$ a.u.. The smallest vector $\mathbf{p}_{\mathrm{e}}$ that we can access is now $p_{\mathrm{e}}=(-1.22,0,0)$ a.u. (i.e. $\left.p_{\mathrm{e}}=G_{\overline{2} 00}\right)$ and its contribution is at $\mathbf{p}_{\text {obs }}=-1.22$ a.u. This is near the edge of the spectral function where the momentum density drops to zero. Thus due to the diffraction by a $[2,2,0]$ reciprocal lattice vector we can expect to observe some extra intensity at $p_{\text {obs }}=-1.22$ a.u. At $\mathbf{p}_{\mathrm{e}}=(-1.22,0,0)$ and $\mathbf{p}_{\mathrm{e}}=$ $(0,-1.22,0)$ the binding energies are identical and hence the diffracted contribution coincides in energy with the direct (without diffraction) contribution. Away from $p_{\text {obs }}=$ -1.22 a.u. the $[2,2,0]$ diffracted intensity hardly contributes, as there the corresponding $\mathbf{p}_{\mathrm{e}}$ vectors are in a region with very low momentum densities.

The next reciprocal lattice vector by which the incoming beam can diffract is of the form $[ \pm 4,0,0]$ and $[0, \pm 4,0]$. The first one shifts the measurement line from $\mathbf{p}_{\mathrm{e}}=$ $(\mp 2.44,-5,0)$ a.u. to the line $\mathbf{p}_{\mathrm{e}}=(\mp 2.44,5,0)$ a.u. The minimum distance of the shifted measurement line to the origin is 2.44 a.u. and thus the momentum density along this line is negligible. Hence, none of the observed coincidence events are associated with diffraction events of type $[ \pm 4,0,0]$. This is not true for $[0,4,0]$ type diffraction events. Now the measurement line extends from $\mathbf{p}_{\mathrm{e}}=$ $(0,(-5-2.44), 0)$ to $\mathbf{p}_{\mathrm{e}}=(0,(5-2.44), 0)$ a.u. This line passes through the origin and thus we can measure even electrons with $\mathbf{p}_{\mathrm{e}}=0$. However, the $\mathbf{p}_{\mathrm{e}}=0$ related intensity appears not at $p_{\text {obs }}=0$ a.u. but at $p_{\text {obs }}=-2.44$ a.u. Thus this diffracted beam causes a (weak) replication of the main spectrum, shifted by 2.44 a.u. The primary (non-diffracted) and $[0,4,0]$ diffracted contributions are well separated everywhere, except near $p_{\text {obs }}=-1.22$ a.u. Similar arguments apply for the $[0,-4,0]$ vector.

Now consider the diffraction of the outgoing electrons. For the measurement with the $[0,1,0]$ crystal direction aligned with the spectrometer $y$-axis the outgoing electrons have trajectories along $[1,0,1]$ and $[-1,0,1]$ direction. The shortest reciprocal lattice vector perpendicular to $[1,0,1]$ is of the type $[1,1,-1]$ (see Fig. 3). Its magnitude (1.06 a.u.) is smaller than any of the reciprocal vectors that affect the incoming beam. Hence, it is more difficult to separate the contribution of this diffraction vector from the clean events. After this type of diffraction, the line along which $\mathbf{p}_{\mathrm{e}}$ is measured extends from $(0.61,(-5+0.61),-0.61)$ a.u. to $\mathbf{p}_{\mathrm{e}}=(0.61,(5+0.61),-0.61)$ a.u. The shortest distance of this line to the origin is at $p_{\mathrm{obs}}=0.86$ a.u. and near this point we expect the maximum binding energy of its contribution. At $p_{\mathrm{obs}}=0$ and $p_{\mathrm{obs}}=-1.22$ a.u. the observed intensity corresponds to that of a $\Gamma$ point $([1,1,-1]$ and $[1,-1,-1]$, respectively) and the observed diffracted intensity will be proportional to $\left|c_{(1,1,1)}\right|^{2}$ times the diffracted beam intensity. This is the only diffraction event that can cause intensity at $p_{\mathrm{obs}}=0$. The intensity at $p_{\mathrm{obs}}=-1.22$ is now due to two contributions: one of the non-diffracted beam is proportional to its intensity times $\left|c_{(0,2,0)}\right|^{2}$ and the one of the diffracted beam is proportional to the diffracted intensity times $\left|c_{(1,1,1)}\right|^{2}$. Both contributions appear at the same binding energy as they are part of the same solution of the Hamiltonian.

In the experiment, it is possible to rotate the sample along the $y$-axis (see Fig. 1(c)). This will not affect the direction along which we measure the energy-resolved momentum densities. However, it will affect the direction of the incoming and outgoing electrons in the coordinate system defined by the crystal lattice and hence their diffraction. The lowering of the symmetry caused by such a rotation will generally mean that fewer 'short' reciprocal lattice vectors are almost perpendicular to the incoming and/ or outgoing electron trajectories. Hence a decrease in the importance of diffraction effects is expected. However, this rotation will not affect the reciprocal lattice vector of the form $[0,2 i, 0]$. These reciprocal lattice vectors will always be perpendicular to the incoming and outgoing beams and its diffraction cannot be avoided. In the current set-up (and possibly in any conceivable EMS setup) it is not possible to measure the momentum densities along a high-symmetry direction and completely avoid diffraction conditions.

A similar analysis can be done for measurements with the $y$-axis of the spectrometer aligned with a $\langle 1,1,0\rangle$ direction of the crystal (see Fig. 1(d)). For the incoming beam aligned with the surface normal, the outgoing electrons propagate now along the $\langle 1 / 2 \sqrt{2},-1 / 2 \sqrt{2}, 1\rangle$ and $\langle-1 / 2 \sqrt{2}, 1 / 2 \sqrt{2}, 1\rangle$ crystallographic directions and the shortest possible diffraction vector is now $\pm<2,2,0\rangle$ for both the incoming and outgoing electrons. In this 
orientation it is not possible to have intensity due to diffracted beams at $p_{\text {obs }}=0$. Diffraction due to $G_{(2,2,0)}$ reciprocal lattice vector cannot be minimised by rotating along the $y$-axis as this vector is parallel to this axis. Thus again diffraction effects due to reciprocal lattice vector pointing along the measurement direction cannot be avoided.

\section{Experimental results}

Experimental momentum densities for two major symmetry directions are presented in Fig. 4. The same normalisation factor is used within each panel, but intensities in the left and right panel cannot be compared directly. The data shown are the raw data and are not corrected for inelastic multiple scattering contributions. In the case of the $\langle 1,0,0\rangle$ axis aligned with the spectrometer's $y$-axis (right panel) we see a peak of relatively constant intensity that disperses upwards by $\simeq 12 \mathrm{eV}$ and near 1 a.u. reaches the top of the valence band. In contrast, if one aligns the $\langle 1,1,0\rangle$ direction with the spectrometer's $y$-axis there is

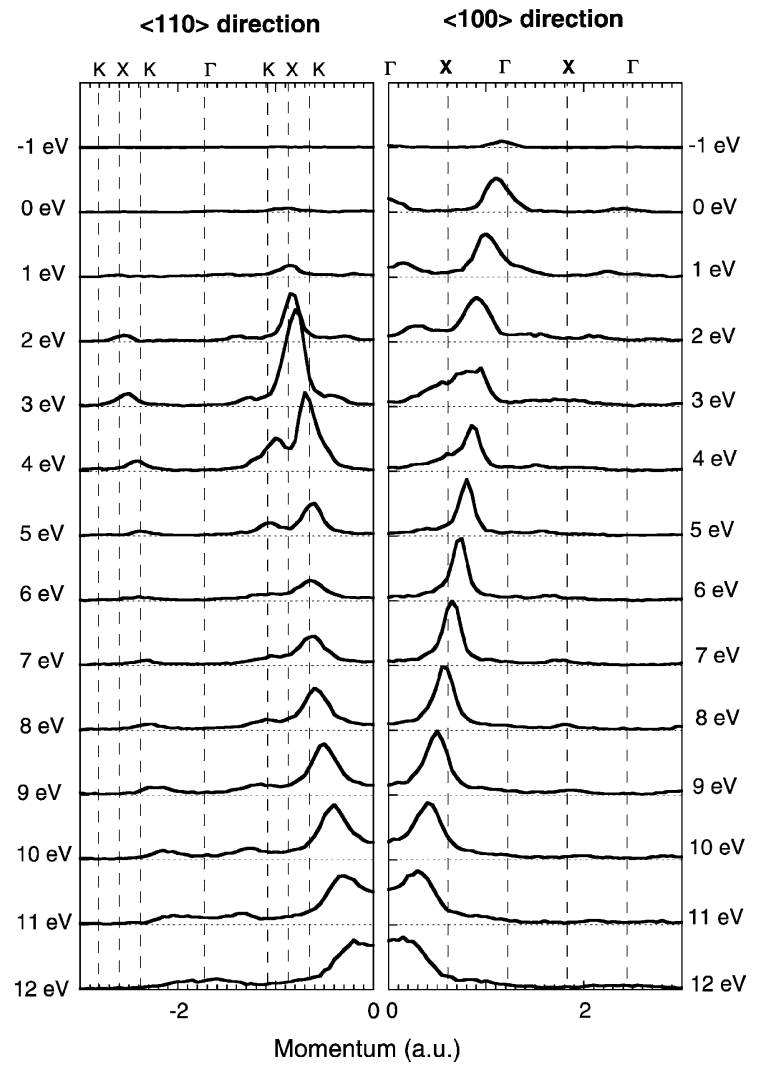

Fig. 4. Measured momentum densities along the $\langle 1,0,0\rangle$ (left) and $\langle 1,1,0\rangle$ direction (right). Momentum densities corresponding to different binding energies, as indicated, are plotted with a vertical offset. The momenta corresponding to major symmetry points of the Brillouin zone are indicated by dashed lines. a profound minimum in intensity at $6 \mathrm{eV}$ below the top of the band and the main intensity increases with increasing momentum (decreasing binding energy) but dropping off in intensity before it reaches 1 a.u.. The minimum binding energy, which is approximately $2 \mathrm{eV}$ larger than the minimum binding energy in the $\langle 1,0,0\rangle$ alignment, is reached at the $X$ point. All these observations are in agreement with the band structure calculations (see Fig. 2).

In Fig. 5 we show the measured energy-resolved momentum densities as a grey-scale plot, with superimposed on it the band structure presented in the repeated zone scheme. Many branches of the calculation, but not all, are reflected in the measurement. For example, as expected, the main structure shifted by the $[0,2,0]$ reciprocal lattice vector, is missing. Along the $\langle 1,1,0\rangle$ direction, the highest intensity is near the point $X$. This is not expected from the band structure calculation. The diffraction related intensity that extends from $X$ to the second $\Gamma$ point may also contribute to this maximum.

In the $\langle 1,1,0\rangle$ measurement there are weaker features, shifted by a reciprocal lattice vector, that mirror the main feature. These are due to diffraction vectors directed along the spectrometer $y$-axis. In the $\langle 1,0,0\rangle$ direction the main 'extra' feature disperses back to zero momentum at the top of the valence band. As explained, these are caused by the outgoing electrons diffracting by $\langle 1,1,1\rangle$ type reciprocal lattice vectors.

In these measurement some of the momenta we accessed correspond to $\Gamma$ points of the reciprocal lattice. All these data are presented in Fig. 6 . For the cases with the $y$-axis of the spectrometer aligned with either the $\langle 1,1,0\rangle$ or $\langle 1,0,0\rangle$ direction we show in Fig. 6 the spectra at $p_{\text {obs }}=0$ (upper panel). Without diffraction both spectra should be equal. At first sight it is therefore somewhat surprising that the two results are qualitatively different. For the $\langle 1,0,0\rangle$ direction we observe two peaks, one corresponding to the bottom of the band, and one corresponding to the top of the band. For the $\langle 1,1,0\rangle$ direction there is only one peak, corresponding to the bottom of the band. This is the peak we expect at zero momentum. From our discussion of diffraction it is clear that for $\langle 1,0,0\rangle$ alignment we can have diffraction with the shortest reciprocal lattice vector $\langle 1,1,1\rangle$. It is this, and only this diffraction vector, that can cause diffracted intensity to show up at $p_{\mathrm{obs}}=0$. The absence of the low binding energy peak in the case of $\langle 1,1,0\rangle$ orientation, unambiguously shows that the upper peak in the $\langle 1,0,0\rangle$ case is due to diffraction and not to a significant contribution of $c_{(0,0,0)}$ to the wave function at the top of the valence band.

For the $\langle 1,0,0\rangle$ orientation, the next $\Gamma$ point is reached at $p_{\text {obs }}=1.22$ a.u. Here we see only the low binding energy feature. This feature is expected for two reasons: nondiffractive $(e, 2 e)$ events will give an intensity proportional to $\left|c_{(0,2,0)}\right|^{2}$ which is $\neq 0$ for the top of the valence band. In addition diffracted beams could produce intensity here proportional to their strength and $\left|c_{(2,2,0)}\right|^{2}$. One should thus be cautious in the interpretation of the magnitude of 

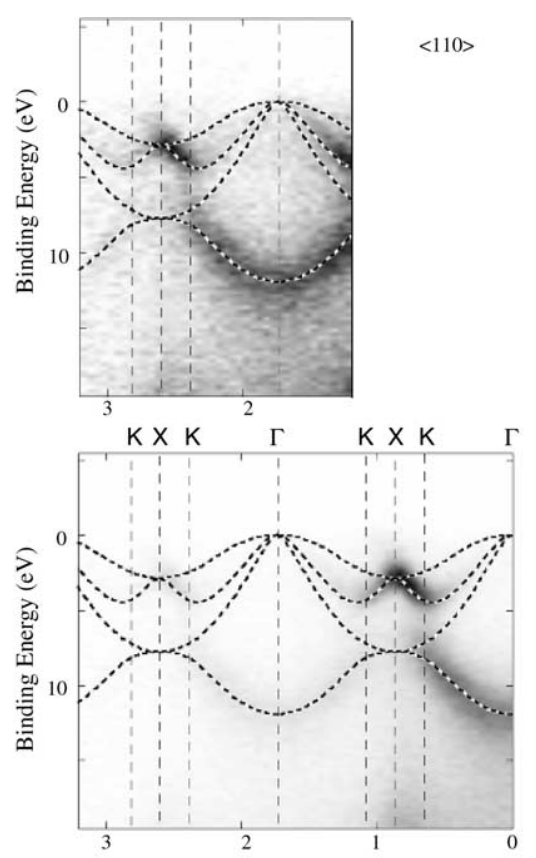

$<110>$
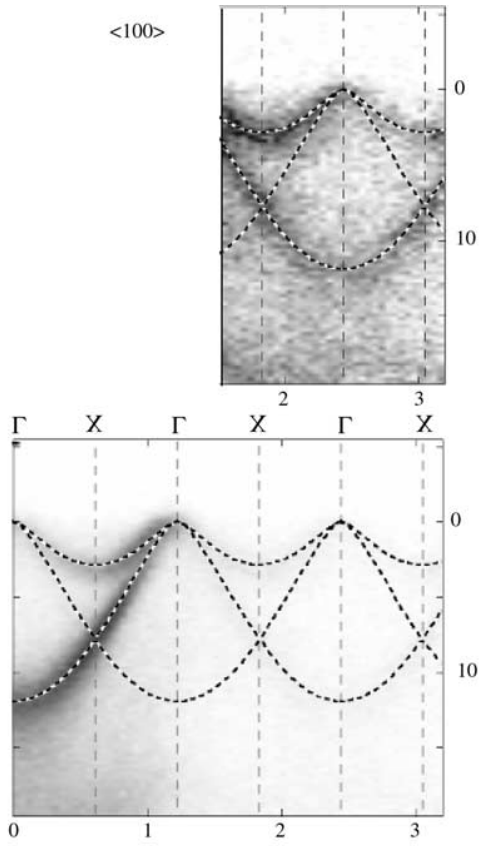

Momentum (a.u.)

Fig. 5. Measured momentum densities along the $\langle 110\rangle$ (left) and $\langle 100\rangle$ direction (right). Measurements are presented as a linear grey-scale intensity plot with the band structure in the repeated zone scheme superimposed. The high momentum part of each plot is repeated at the top, with an adjusted grey-scale that emphasises the structure present in the low intensity part of the distribution.

the momentum density at this momentum value, since it contains two contributions. The high binding energy feature does not show up at $p_{\text {obs }}=1.22$ a.u., as the diffracted beam with $G_{(0,2,0)}$ has zero intensity for silicon. At the next $\Gamma$ point (at $p_{\text {obs }}=2.44$ a.u.) the measured spectrum is to a good approximation a (weaker) replication of the $p_{\mathrm{obs}}=0$ momentum spectrum.

In another measurement the crystal was rotated along the surface normal in such a way that a $\langle 1,1,0\rangle$ crystallographic direction coincided with the spectrometer $y$-direction (left hand panel of Fig. 6). The single diffraction feature here is that associated with $G_{(2,2,0)}$. Only a single peak is seen at $p_{\text {obs }}=0$ corresponding to maximum binding energy. At the next $\Gamma$ point $\left(p_{\text {obs }} \simeq 1.7\right.$ a.u. $)$ the spectrum is again a replication of the spectrum at zero momentum. The absence of the low binding energy peak at 1.7 a.u. shows that $\left|c_{(2,2,0)}\right|^{2}$ is indeed small for the wave function at the top of the valence band. Moreover, diffracted contributions of

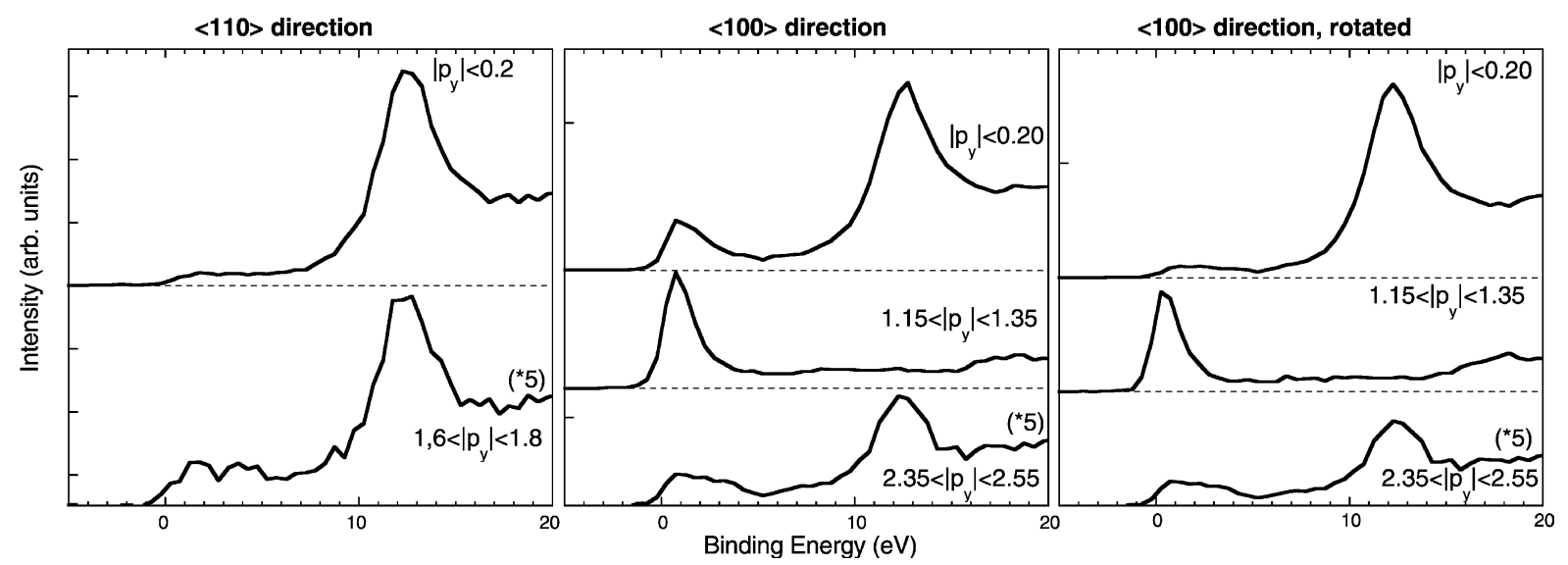

Fig. 6. Eight spectra summed over momentum bins of 0.2 a.u. taken under different conditions, all corresponding to $\Gamma$ points. The $y$-axis of the spectrometer is aligned with either the $\langle 1,1,0\rangle$ direction (left panel), or the $\langle 1,0,0\rangle$ direction (central and right panels). In the right panel the sample was rotated $10^{\circ}$ around the $y$-axis and the incoming and outgoing electron trajectories are not along major crystallographic directions. The intensities of the lowest panels have been multiplied by a factor of 5 . 
the upper valence band feature do not show up as no reciprocal lattice vectors that connect $G_{(2,2,0)}$ with $G_{(0, \pm 2,0)}$ or $G_{(1,1,1)}$ are perpendicular to either the incoming or outgoing beams.

For the measurement of the $\langle 1,0,0\rangle$ direction we also rotated the sample $10^{\circ}$ around the spectrometer $y$-axis, to test the prediction that this lower symmetry configuration would reduce the diffraction effects (see Fig. 6, right panel). Indeed, the low binding energy structure for the spectrum at $p_{\text {obs }}=0$ a.u. has now essentially disappeared. Thus a $10^{\circ}$ rotation is enough to suppress the diffraction by vectors of type $\langle 1,1,1\rangle$ by at least an order of magnitude. The shapes of the second and third $\Gamma$ spectra were not affected by the rotation. The fact that the intensity of the third $\Gamma$ spectrum (at $p_{\text {obs }}=2.4$ a.u.) has not changed greatly is expected, as the direction of the $\langle 0,4,0\rangle$ reciprocal lattice vector is not affected by the rotation, and remains perpendicular to the incoming beam.

Note that there is still significant intensity at the high binding energy side of the quasi-particle peaks. This is in part due to inelastic multiple scattering and in part due to plasmaron-type intrinsic satellites. Disentanglement of these contributions will be discussed in a separate paper [15].

\section{Discussion and conclusion}

In the preceding sections we gave a qualitative description of the measured energy - momentum densities in terms of diffraction. We treated the effect of diffraction as merely changing the direction of propagation of either the incoming or outgoing electrons. In reality we have to consider the phase of the primary and diffracted beam. Their well defined phase relation means that interference between these two beams causes standing waves to appear in the crystal. Schülke et al. [16] and Golovchenko et al. [17] have shown that for the case of X-rays the spatial position of the nodes can significantly change the probability that core electrons contribute to Compton profiles. For the case of electron Compton scattering this effect was confirmed by Williams and Bourdillon [18]. In principle, the $(e, 2 e)$ measurements with better controlled diffraction conditions could do the same for the valence band. The least bound valence electrons are generally pictured as standing waves with large amplitude at the nuclei (area of large potential energy) whereas the lowest unoccupied state, with the same wave number, has nodes at the nuclei. This simple text-book picture could, at least in principle, be experimentally verified by EMS. Changing diffraction conditions should change the intensity of the peaks at the top of the valence band. Monitoring simultaneously the intensity of the $\mathrm{Si} 2 \mathrm{p}$ core level and that of the outer valence band would reveal if the dependency of their intensities on the diffraction condition is similar. This would be an experimental verification of the aforementioned simple text-book picture.
All data shown here were symmetrised, i.e. we added the measured intensity at $\pm p_{\text {obs }}$. In this way we improved statistics. The binding energy positions of peaks at $\pm p_{\mathrm{obs}}$ were always identical, but the intensities were not, most notably for the diffracted peaks: these could be 2-3 times stronger at positive momentum compared to negative momentum or vice-versa. Experimentally there is no reason for this deviation, and it is most likely caused by a slight tilt around the $x$-axis of the sample. In order to compare these spectra with full-fledged calculations based on the dynamical theory of diffraction we are making alterations to control this orientation. Further investigations would also require that we establish the thickness of the sample more quantitatively.

In summary, we have shown that diffracted EMS intensity can often be distinguished from 'direct' intensity. Changing the diffraction conditions for the incoming and/or outgoing electrons will pinpoint which part of the intensities is diffraction related. In practice diffraction is often minimised by lowering the symmetry of the configuration. This rule can be rephrased to state that for the (most interesting) high-symmetry directions, diffraction cannot be avoided completely. For materials such as silicon with only a few valence electrons per unit cell, diffraction contributions are usually well separated from the direct intensity, and ambiguities are restricted to certain specific parts of the valence band. Testing the theory by comparing measurements and calculations along well defined low symmetry directions could be a fruitful way of comparing theory and experiment without ambiguities due to diffraction.

\section{Acknowledgements}

The authors want to thank Dr L.J. Allen for carefully reading the manuscript. We also acknowledge the Australian Research Council for providing financial support.

\section{References}

[1] S. Hüfner, Photoelectron Spectroscopy, Springer, Berlin, 1995.

[2] E. Weigold, I.E. McCarthy, Electron Momentum Spectroscopy, Kluwer Academic/Plenum, New York, 1999.

[3] M. Vos, I.E. McCarthy, Observing electron motion in solids, Rev. Mod. Phys. 67 (1995) 713-723.

[4] M. Vos, Z. Fang, S. Canney, A.S. Kheifets, I.E. McCarthy, E. Weigold, Electron-momentum density of graphite by $(\mathrm{e}, 2 \mathrm{e})$ spectroscopy, Phys. Rev. B 56 (1997) 963-966.

[5] M. Vos, A.S. Kheifets, V. Sashin, E. Weigold, Anisotropy and correlation effects in the spectral function of graphite as measured by electron momentum spectroscopy, AIP Conference Proceedings 652 (2003) 491-496.

[6] Z. Fang, R.S. Matthews, S. Utteridge, M. Vos, S.A. Canney, X. Guo, I.E. McCarthy, E. Weigold, Electron momentum spectroscopy on crystal silicon, Phys. Rev. B 57 (1998) $12882-12889$ 
[7] V.A. Sashin, S.A. Canney, M.J. Ford, M.A. Bolorizadeh, D.R Oliver, A.S. Kheifets, Energy-resolved momentum densities for the valence band of a nanoscale Si single crystal, J. Phys.: Condens. Matter 12 (2000) 125-136.

[8] M. Vos, G.P. Cornish, E. Weigold, A high-energy (e,2e) spectrometer for the study of the spectral momentum density of materials, Rev. Sci. Instrum. 71 (2000) 3831-3840.

[9] L.J. Allen, I.E. McCarthy, V.W. Maslen, C.J. Rossouw, Effects of diffraction on the (e,2e) reaction in crystals, Aust. J. Phys. 43 (1990) 453-464.

[10] R. Matthews, $\mathrm{PhD}$ thesis, Flinders University of South Australia, 1993.

[11] S.J. Utteridge, V.A. Sashin, S.A. Canney, M.J. Ford, Z. Fang, D.R. Oliver, M. Vos, E. Weigold, Preparation of a $10 \mathrm{~nm}$ thick single-crystal silicon membrane self-supporting over a diameter of $1 \mathrm{~mm}$, Appl. Surf. Sci. 162-163 (2000) 359-367.

[12] M. Vos, E. Weigold, Developments in the measurement of spectral momentum densities with $(e, 2 e)$ spectrometers, J. Electron Spectrosc. Relat. Phenom. 112 (2000) 93-106.

[13] A.S. Kheifets, D.R. Lun, S.Y. Savrasov, Full-potential linearmuffin-tin-orbital calculation of electron momentum densities, J. Phys.: Condens. Matter 11 (1999) 6779-6792.

[14] N.W. Ashcroft, N.D. Mermin, Solid State Physics, HoltSaunders, 1976.

[15] A. Kheifets, V. Sashin, M. Vos, E. Weigold, F. Aryasetiawan, Spectral properties of quasiparticles in silicon: a test of manybody theory, unpublished.

[16] W. Schülke, U. Bonse, S. Mourikis, Compton scattering at the Bragg position, Phys. Rev. Lett. 47 (1981) 1209-1212.

[17] J.A. Golovchenko, D.R. Kaplan, B. Kincaid, R. Levesque, A. Meixner, M.F. Robbins, J. Felsteiner, Coherent Compton effect, Phys. Rev. Lett. 46 (1981) 1454-1457.

[18] B.G. Williams, A.J. Bourdillon, Localised Compton scattering using energy-loss spectroscopy, J. Phys. C: Solid State Phys. 15 (1982) 6881-6890. 